\title{
Asynchronous Validation and Documentation of Sterile Compounding in a Hospital Pharmacy
}

\author{
Denis Lebel, Maxime Thibault, and Jean-François Bussières
}

\section{INTRODUCTION}

$\mathrm{T}$ The US Pharmacopeial Convention published the USP $1<797>$ Guidebook to Pharmaceutical CompoundingSterile Preparations in 2008. ${ }^{1}$ This publication, which is based on the USP Revised General Chapter <797>, sets practice standards to help ensure that compounded sterile preparations are of high quality. Although these guidelines have not yet been enforced by the Quebec regulatory authority, they will be the basis of revised guidelines to be published in 2011 by l'Ordre des pharmaciens du Québec. The release of the USP Revised General Chapter $<797>$ is prompting pharmacists and technicians to substantially revise their practice to reduce potential contamination, to limit access to the sterile zone whenever possible, and to appropriately document all steps of the compounding process.

In our institution, pharmacy technicians prepare sterile products several times a day. Each of these products must be verified by a pharmacist, which entails the pharmacists entering and exiting the clean room repeatedly, in addition to carrying out their other duties. This time-consuming practice increases the risk of microbiological and particulate contamination. Furthermore, each visual inspection performed in the clean room interrupts the workflow of both the pharmacist and the technician. Interruptions and distractions are a documented cause of medication errors in pharmacies. ${ }^{2,3}$

Traditionally, telepharmacy ${ }^{4}$ has been defined as the use of various telecommunications systems to provide pharmaceutical services and care to remote or rural areas ${ }^{5-12}$ or to provide such services during times when pharmacists are not readily available on site (e.g., at night). ${ }^{13-16}$ When the photographic system described in the current article was being developed, there were no published reports on the use of cameras as an aid during sterile compounding.

We analyzed our procedures and set the following objectives: (1) to develop an asynchronous workflow that would reduce interruptions (and thereby reduce the risk of medication errors) and (2) to develop a technological solution that would enable pharmacy technicians to capture images of the various steps in preparing medications, which would in turn allow the pharmacist to asynchronously verify the accuracy of the pharmaceutical ingredients, materials, and quantities and would serve as documentation for subsequent reference. This article describes the development, implementation, and use of a photographic system that allows pharmacists to supervise and document the activities of pharmacy technicians according to a time-delayed approach.

\section{METHODS}

Because the primary objective of this project was to minimize interruptions, information captured by the camera system had to be accessible in a time-delayed fashion so that pharmacy technicians would not have to wait for the pharmacists' verification to continue their work and so that pharmacists could complete, without distraction, any tasks already in progress. We were therefore obliged to reject video systems, such as closed-circuit television and capture of a video feed, because review of video files to validate pharmaceutical preparations would take too long; in addition, video files consume excessive memory space and cannot be quickly manipulated through a computer network. These systems seem more appropriate for synchronous supervision. In contrast, we wanted the pharmacists to be able to access images from the camera system directly at their computer, while simultaneously accessing the associated pharmaceutical files, to link pictures of preparations with the specific medication orders. Our paperless pharmacy system already offered the ability to link orders to files (e.g., scanned-in faxes) available on the network. We therefore considered only cameras that could feed directly into the hospital's network without the need for additional computers or devices. 
Finally, the pictures obtained had to be clear and detailed enough for pharmacists to verify lot numbers and expiry dates on drug packaging (details that often appear in very small characters) and volumes of liquids in syringes.

\section{Work Setting}

We were reluctant to install the cameras directly in our laminar-airflow workbenches, out of concern that they would cause turbulence and their presence would be problematic for technicians. Moreover, placing cameras inside the workbenches would have necessitated regular disinfection to comply with quality assurance procedures and ensure an ISO class 5 environment within the workbenches. ${ }^{17}$ We often used ethanol, chlorhexidine, and sometimes sodium hypochlorite (bleach) for disinfection, and these agents might have hastened the deterioration of certain sensitive parts of the cameras, such as their lenses, if the cameras were placed inside the workbenches. We therefore installed each camera on an articulated arm outside the workbench, with the lens adjusted to focus slightly above the work surface. Objects placed in the centre of this area were automatically in focus. We also installed a computer and monitor outside each laminar-airflow workbench, so that the technicians could view the camera feed at all times and thus ensure that the objects in the picture were in focus for easy viewing later on by the pharmacists.

\section{DESCRIPTION OF SYSTEM}

\section{Camera and Lens}

We selected IQeye 702 cameras (IQinVision, San Juan Capistrano, California) with 2-megapixel resolution. These cameras use technology compliant with IEEE 802.3AF Powerover-Ethernet standards. As such, power for the cameras comes directly through the Ethernet cable that connects them to the computer network, which reduces the need for additional wires. The cameras also have an integrated web server, which simplifies image acquisition (the images being transmitted over the hospital network). We chose 25-mm lenses that could capture images with details in a wide range of sizes, from expiry dates on small vials to larger characters on $500-\mathrm{mL}$ bags. We selected lenses without autofocusing capability, to limit problems with focusing through the glass of the hood, especially in the presence of reflected light. Similarly, we chose cameras without pan-tilt-zoom technology. That capability would have allowed the pharmacist to pan, tilt, or zoom the images at will, but would have necessitated active monitoring of the image-capture procedure, and these adjustments would have interrupted the technicians' work.

\section{Articulated Arm}

In consultation with the hospital's metal workers, we developed a custom system to anchor the articulated arms to the laminar-airflow workbenches without the need to drill any holes in the workbenches. To each anchor, we affixed an Ergotron computer support system (Ergotron, Saint Paul, Minnesota) and 2 Ergotron articulated arms, one to support the computer's LCD (liquid crystal display) screen and the other to support the camera. Ergotron did not offer a cameramounting system, so we designed our own adapter, an L-shaped metal piece, with one part fixed to the arm piece of the flat-screen monitor and the other carrying a standard screw of the type used for tripods.

\section{Image Flow and Network Storage}

Image acquisition was controlled by a foot pedal connected via a USB port to the computer installed on the laminar-airflow workbench. Triggering the pedal saved the image being displayed by the camera's video feed to a shared network drive. A program run by the server hosting this shared drive scanned the drive every minute, moving any new pictures found to a secure database, from which the picture entered the queue module of the pharmacy information system. The module displayed pictures in chronological order. A pharmacist could then permanently link a specific picture to an order in a patient's file, using a method that associated the image's unique identifying number with the order's unique identifying number in a data table. The pharmacists could then retrieve pictures related to the preparation of a given order whenever necessary. Figure 1 summarizes the flow of information through the network. Once the images had been linked to a specific order, a pharmacist validated the preparation by verifying 3 of the 4 elements required for appropriate compounding: proper components, proper quantities, and proper labelling. To ensure that the pharmacist could look at a single picture and validate the preparation in one step, each image had to show the pharmacy's label for the medication (identifying both the patient and the medication), as well as any other elements that would usually require verification by a pharmacist (e.g., liquid volumes measured in syringes, source containers for drugs, batch numbers and expiry dates, diluents, and final packaging). The pharmacists did not actively verify the fourth element of compounding, the technician's preparation technique, which was covered through a separate training program.

\section{Software}

A pharmacist with appropriate training and knowledge designed and wrote an image-capture program with USB footpedal support. The software was connected to the camera's web server and displayed the current video feed on an embedded web page. When the pedal was depressed, the program captured a high-resolution still $(1600 \times 1600$ pixels $)$ of the camera's video feed and saved it as a JPEG image on the shared network drive. The program also displayed a full-screen version 


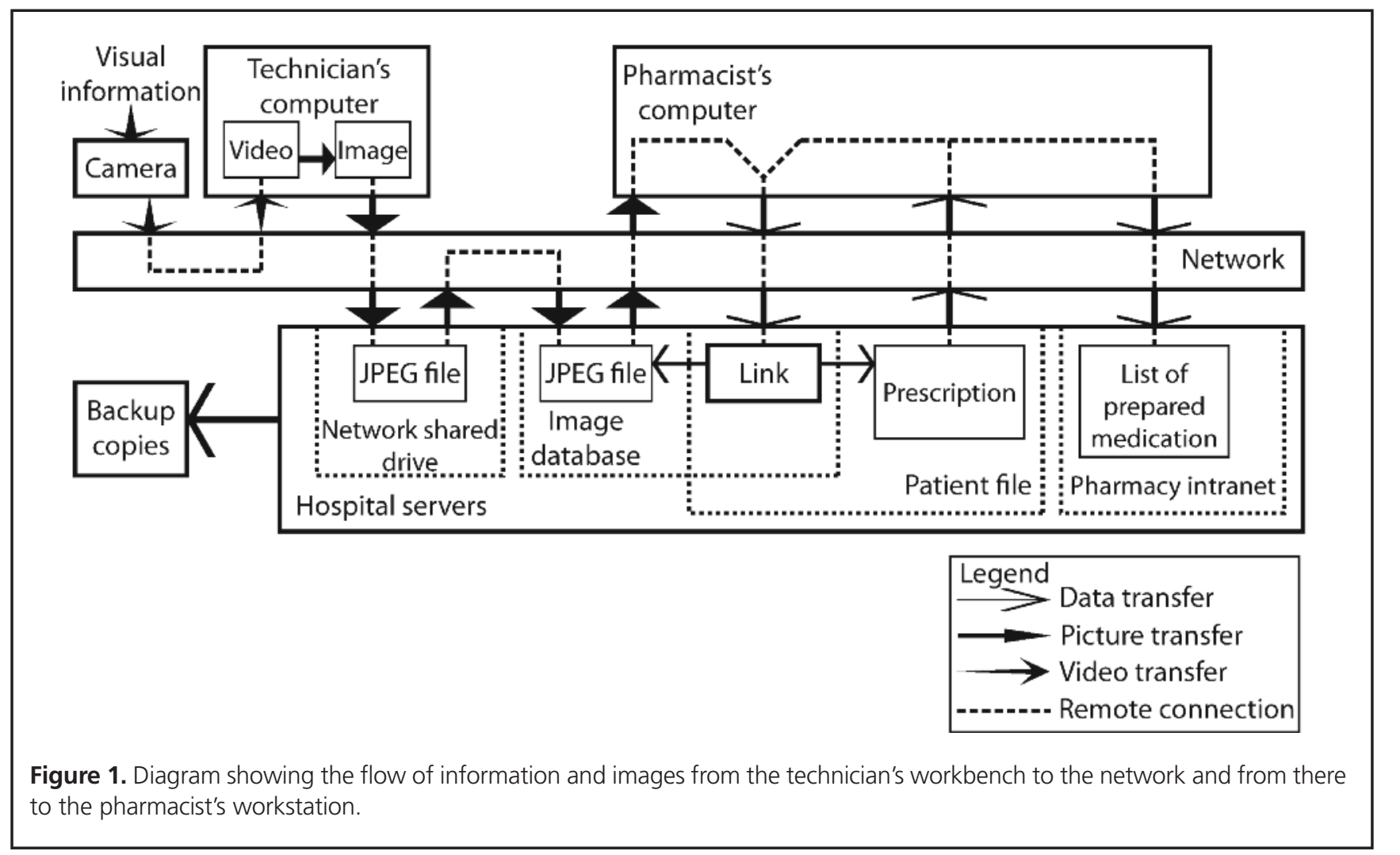

of each image on the pharmacy technician's 19-inch (48.2-cm) monitor for 15 seconds.

NumeRx, a paperless module of GesphaRx software (CGSI Solutions TI Inc, Québec, Quebec), was then used to retrieve the images from the temporary folder, store them permanently in a database, display them in the queue, and manage associations with the drug order. Although paperless modules of pharmacy information software are often used for faxed or digitized orders, use of this module to process images and document pharmaceutical preparations was innovative.

\section{Utilization by Technicians}

As noted previously, the technician could at all times see the camera's video feed on his or her computer screen and could thus ensure that all required elements were in focus within the picture. Interaction with the system consisted solely of pressing the foot pedal to take a picture. This prevented the microbiological or particulate contamination that might have occurred if use of a keyboard or mouse had been required. Once the picture had been taken, the technician could proceed to finish the preparation without waiting for a pharmacist's approval; any error would be identified asynchronously during subsequent verification by the pharmacist. This met the objective of minimizing workflow interruptions for both technicians and pharmacists. Exceptionally, for very time-consuming or expensive preparations, in which an error would cause excessive loss of time or money, technicians could ask for immediate verification through a live video feed, which a pharmacist could watch on the camera's web page. Figure 2 shows the physical setting of the clean room and a typical view from the technician's perspective.

\section{Utilization by Pharmacists}

The set-up of the pharmacists' workspace ensured that only preparations with appropriate validation could be dispensed. Each computer workstation had 3 LCD screens: one for the pictures taken by the technicians' cameras, a second for the medication profile, and a third used by specialized software that displayed "ready" status to the wards once a pharmacist had scanned each preparation's bar code (Figure 3). Nurses and clerks on the wards had access to the list of scanned medications and could see when their patients' medications were ready.

\section{RESULTS}

\section{Effect on Work Interruptions}

At the time of writing, in late 2009, this system had been in use in the oncology pharmacy of our hospital for 28 months, and a total of 51278 images had been obtained on 467 workdays. Assuming that every picture represented one less interruption, and given the standard 8-h workday (0800 to 1600), we estimated that the system prevented 13.72 interruptions per hour of work. We believe that this represented a 


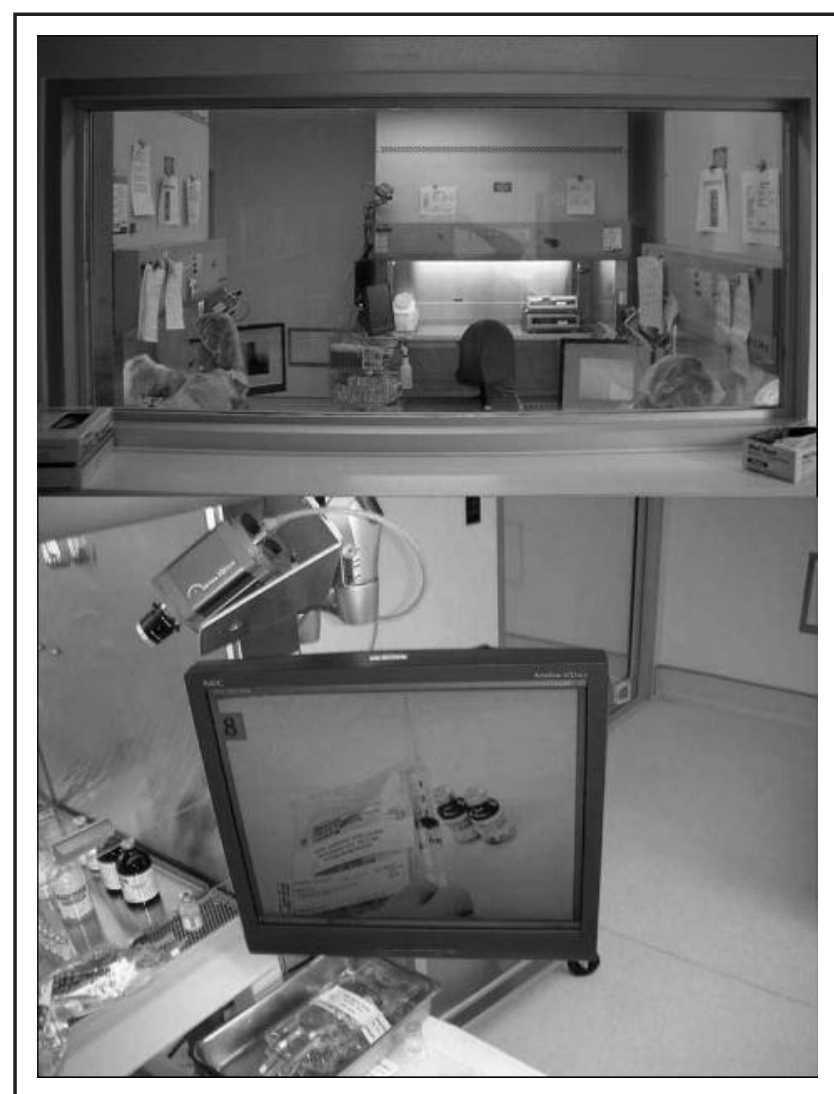

Figure 2. Set-up of the camera in the clean room of the pharmacy's oncology satellite unit, showing a typical view from the technician's perspective.

substantial reduction in distractions for both technicians and pharmacists, a crucial factor in preventing medication errors.

\section{Effect on Patient Safety}

We developed this system to reduce the impact of moving into a new USP Chapter <797>-compliant pharmacy. In particular, the use of a double airlock, as required by Chapter $<797>$, greatly limited pharmacists' ability to combine supervision of dose preparations with other duties. By implementing the camera system, we were able to implement procedures complying with the USP Chapter $<797>$ standards without any cuts to our clinical services. In addition, reducing interruptions was an important step in improving patient safety. Visual documentation of preparations was also an advantage.

As one example of the benefits of the new system, a pharmacist in the oncology unit recently reported that 3 patients had had significant adverse effects (seizures) after receiving a certain treatment. Using the image database, we were able to trace the product used and confirm that the manufacturer's lot number was the same for all 3 patients. This allowed us to inform the drug manufacturer of a potential problem with that specific batch.

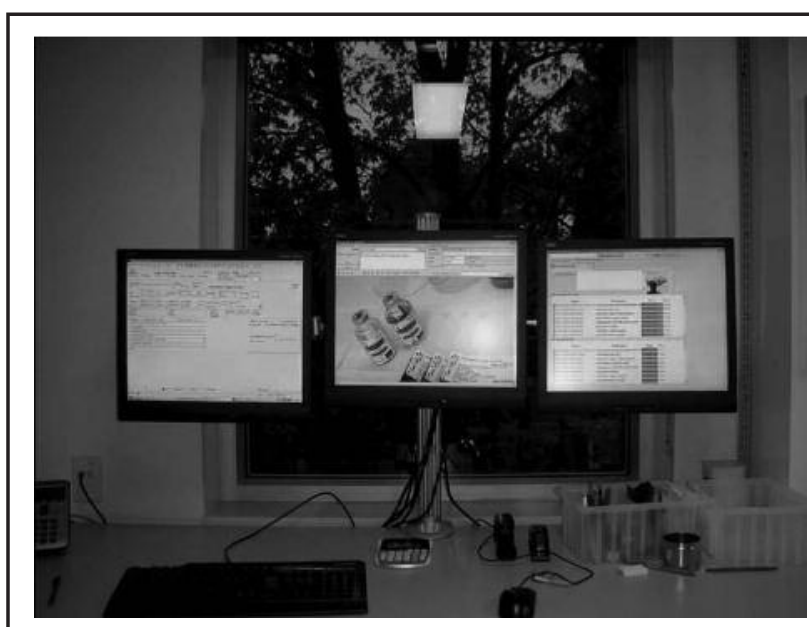

Figure 3. The pharmacists' computer workstation, with 3 screens: one for the pictures taken by the technicians' cameras, a second for the medication profile, and a third to display "ready" status to the wards.

\section{Problems Encountered}

We experienced problems with deposition of particles on the camera sensors. These particles distorted the images captured. In addition, a crack developed in the sensor of one camera during the first few weeks of testing.

Because this system relies heavily on the hospital's network infrastructure, it is conceivable that network issues could affect its operation. We encountered a few minor problems relating to connectivity during the test phase, but as of the time of writing, the situation was stable. The hospital's network (gigabit Ethernet) has no difficulty handling the data flow generated by the images and video feeds. The system has been running for many months with only minor interruptions related to connectivity.

\section{DISCUSSION}

The new camera system had a positive impact on workflow in the oncology pharmacy. The pharmacists and technicians were pleased with the results, and the benefits of the system are observed on a daily basis. In particular, pharmacists and technicians seldom interrupt each other's work for the validation of pharmaceutical preparations.

We compared our system with similar ones used in 2 other hospitals in Quebec, as reported previously. ${ }^{18}$ Both of those systems used closed-circuit television cameras and a live video feed for monitoring technicians' work. Both systems eliminated the need for pharmacists to enter and exit the clean room, but because they did not involve an asynchronous process, they had no impact on work interruptions. However, they did allow pharmacists to supervise the preparation technique, which is not possible with our system. Although one centre initially 
installed cameras directly inside its laminar-airflow workbenches, testing showed that the cameras created turbulence. As a result, the cameras were installed on articulated arms outside the laminar-airflow workbenches. One of the closed-circuit systems, which used pan-tilt-zoom technology, allowed pharmacists to verify batch numbers and expiry dates on product containers. However, the other system did not allow this level of verification, because the resolution of the closedcircuit television was too low without the zoom function. In the latter case, batch numbers and expiry dates were verified when the completed product was transferred outside the clean room, along with every product container used in the preparation.

We also compared our system with one based on Scriptpro hardware and software, described in late 2009 by O'Neal and others. ${ }^{19}$ We believe that the system described here is more flexible and less expensive than the system described by O'Neal's team.

The proof of concept reported here had some limitations. Expected challenges in implementing the technology included the design and installation of the articulated arms on the laminar-airflow workbenches. Each installation had to be evaluated in terms of the specific work environment. Another challenge concerned the archiving and linking of images to the preparation orders. Although these are standard features in paperless pharmacy software programs such as NumeRx, one would need to find a way to tag images for retrieval from the image archive when they are needed.

\section{CONCLUSIONS}

As a result of our positive experience with this technology, we have implemented the system in other compounding areas of the pharmacy. The system has been helpful in several cases, both during audits of the quality of preparations and in analysis of patient safety issues. Our study has demonstrated the potential of a low-cost technology to support asynchronous validation of compounding activities in a hospital pharmacy. Further studies are required to evaluate the full benefits of this type of technology.

\section{References}

1. USP <797> guidebook to pharmaceutical compounding-sterile preparations. Rockville (MD): United States Pharmacopeial Convention.

2. Anacleto TA, Perini E, Rosa MB, César CC. Medication errors and drugdispensing systems in a hospital pharmacy. Clinics (Sao Paulo) 2005; 60(4):325-332.

3. Beso A, Franklin BD, Barber N. The frequency and potential causes of dispensing errors in a hospital pharmacy. Pharm World Sci 2005; 27(3):182-190.

4. Focus group on telepharmacy. Am J Health Syst Pharm 2001;58(2):167-169.

5. Bynum A, Hopkins D, Thomas A, Copeland N, Irwin C. The effect of telepharmacy counseling on metered-dose inhaler technique among adolescents with asthma in rural Arkansas. Telemed $J$ E Health 2001;7(3):207-217.
6. Lordan D, Vorhees N, Richards C. Telepharmacy offers hope for rural hospitals. National pharmacist shortage prompts innovative approach to hospital-based services in Washington state. Telemed Today 2002;9(3):13-15.

7. Clifton GD, Byer H, Heaton K, Haberman DJ, Gill H. Provision of pharmacy services to underserved populations via remote dispensing and two-way videoconferencing. Am J Health Syst Pharm 2003;60(24): 2577-2582.

8. Caceres C, Gomez EJ, Garcia F, Chausa P, Guzman J, Del Pozo F, et al. A home integral telecare system for HIV/AIDS patients. Stud Health Technol Inform 2005;114:23-29.

9. Kimber MB, Peterson GM. Telepharmacy-enabling technology to provide quality pharmacy services in rural and remote communities. $J$ Pharm Res 2006;36(2):128-133.

10. Peterson CD, Rathke A, Skwiera J, Anderson JHC. Hospital telepharmacy network: delivering pharmacy services to rural hospitals. J Pharm Technol 2007;23(3):158-165.

11. Rose JL. Improved and expanded pharmacy care in rural Alaska through telepharmacy and alternative methods demonstration project. Int $J$ Circumpolar Health 2007;66 Suppl 1:14-22.

12. Young D. Telepharmacy project aids North Dakota's rural communities. Am J Health Syst Pharm 2006;63(19):1776,1779-1780.

13. Keeys CA, Dandurand K, Harris J, Gbadamosi L, Vincent J, JacksonTyger B, et al. Providing nighttime pharmaceutical services through telepharmacy. Am J Health Syst Pharm 2002;59(8):716-721.

14. Woodall SC. Remote order entry and video verification: reducing afterhours medication errors in a rural hospital. Jt Comm J Qual Saf 2004; 30(8):442-447.

15. Stubbings T, Miller C, Humphries TL, Nelson KM, Helling DK. Telepharmacy in a health maintenance organization. Am J Health Syst Pharm 2005;62(4):406-410.

16. Boon AD. Telepharmacy at a critical access hospital. Am J Health Syst Pharm 2007;64(3):242-244

17. Kastango ES; American Society of Health-System Pharmacists. Blueprint for implementing USP chapter 797 for compounding sterile preparations. Am J Health Syst Pharm 2005;62(12):1271-1288.

18. Lebel D, Ponton M, Racine MC, Bussières JF. Application pratique de la télépharmacie pour les préparations stériles de médicaments. Pharmactuel 2008;41(1):44-47.

19. O'Neal BC, Worden JC, Couldry RJ. Telepharmacy and bar-code technology in an i.v. chemotherapy admixture area. Am J Health Syst Pharm 2009;66(13):1211-1217.

Denis Lebel, BPharm, MSC, FCSHP, is Assistant Director of the Department of Pharmacy and Research Unit in Pharmacy Practice, Centre hospitalier universitaire Sainte-Justine, Montréal, Quebec.

Maxime Thibault, BPharm, MSC, is a pharmacist with the Department of Pharmacy and Research Unit in Pharmacy Practice, Centre hospitalier universitaire Sainte-Justine, Montréal, Quebec.

Jean-François Bussières, BPharm, MSc, MBA, FCSHP, is Director of the Department of Pharmacy and Research Unit in Pharmacy Practice, Centre hospitalier universitaire Sainte-Justine, Montréal, Quebec.

\section{Address correspondence to:}

Denis Lebel

Department of Pharmacy

Centre hospitalier universitaire Sainte-Justine

3175, chemin de la côte Sainte-Catherine

Montréal QC H3T 1C5

e-mail: denis.lebel.hsj@ssss.gouv.qc.ca 